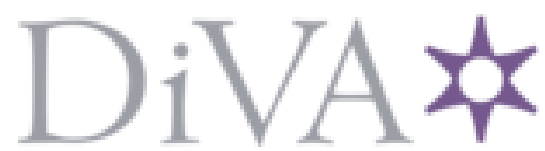

http://www.diva-portal.org

\title{
Postprint
}

This is the accepted version of a paper published in International Journal of Sports Physiology and Performance. This paper has been peer-reviewed but does not include the final publisher proofcorrections or journal pagination.

Citation for the original published paper (version of record):

Lanferdini, F J., Bini, R R., Figueiredo, P., Diefenthaeler, F., Mota, C B. et al. (2016)

Differences in Pedaling Technique in Cycling: A Cluster Analysis..

International Journal of Sports Physiology and Performance, 11(7): 959-964

http://dx.doi.org/10.1123/ijspp.2015-0142

Access to the published version may require subscription.

N.B. When citing this work, cite the original published paper.

Permanent link to this version:

http://urn.kb.se/resolve?urn=urn:nbn:se:gih:diva-4656 


\section{DIFFERENCES IN PEDALLING TECHNIQUE IN CYCLING: A CLUSTER ANALYSIS}

Category of submission: Original Investigation

Fábio J. Lanferdini ${ }^{1}$, Rodrigo R. Bini ${ }^{2}$, Pedro Figueiredo ${ }^{1,3}$, Fernando Diefenthaeler ${ }^{4}$, Carlos B. Mota ${ }^{5}$, Anton Arndt ${ }^{6,7}$ and Marco A. Vaz ${ }^{1}$

${ }^{1}$ Exercise Research Laboratory, School of Physical Education, Physical Therapy and Dance, Federal University of Rio Grande do Sul, Porto Alegre, Brazil

${ }^{2}$ School of Physical Education of the Army, Center of Physical Fitness of the Army, Rio de Janeiro, Brazil

${ }^{3}$ Centre of Research, Education, Innovation and Intervention in Sport, Faculty of Sport, University of Porto, Portugal

${ }^{4}$ Sports Centre, Biomechanics Laboratory, Federal University of Santa Catarina, Florianópolis, Brazil

${ }^{5}$ Center for Physical Education and Sports, Department Sports Techniques and Methods, Federal University of Santa Maria, Brazil

${ }^{6}$ The Swedish School of Sport and Health Sciences, Stockholm, Sweden

${ }^{7}$ Karolinska Institute, Stockholm, Sweden

\section{Corresponding author}

Fábio Juner Lanferdini

Laboratório de Pesquisa do Exercício

Escola de Educação Física

Universidade Federal do Rio Grande do Sul

Rua Felizardo, 750

Porto Alegre, RS, Brasil, CEP 90690-200

Phone 55-51-33085859

Email: fabiolanferdini@gmail.com

Running head: Pedal technique and cycling performance

Abstract Word Count: 252

Text-only Word Count: 4120

Number of Figures and Tables: 0 figures and 3 tables 


\begin{abstract}
The purpose of this study was to employ cluster analysis to assess if cyclists would opt for different strategies in terms of neuromuscular patterns when pedalling at the power output of their second ventilatory threshold ( $\mathrm{PO}_{\mathrm{VT} 2}$ ) compared to cycling at their maximal power output (POMAX). Twenty athletes performed an incremental cycling test to determine their power output ( $\mathrm{PO}_{\mathrm{MAX}}$ and $\mathrm{PO}_{\mathrm{VT2}}$; first session) and pedal forces, muscle activation, muscle-tendon unit length, and vastus lateralis architecture (fascicle length, pennation angle, and muscle thickness) were recorded (second session) in $\mathrm{PO}_{\mathrm{MAX}}$ and $\mathrm{PO}_{\mathrm{VT} 2}$. Athletes were assigned to two clusters based on the behaviour of outcome variables at POVT2 and $\mathrm{PO}_{\mathrm{MAX}}$ using cluster analysis. Clusters \#1 ( $\mathrm{n}=14)$ and \#2 $(\mathrm{n}=6)$ showed similar power output and oxygen uptake. Cluster \#1 presented larger increases in pedal force and knee power than Cluster \#2, without differences for the index of effectiveness. Cluster \#1 presented less variation in knee angle, muscle-tendon unit length, pennation angle and tendon length compared to Cluster \#2. However, Clusters \#1 and \#2 showed similar muscle thickness, fascicle length and muscle activation. When cycling at $\mathrm{PO}_{\mathrm{vT} 2}$ versus $\mathrm{PO}_{\mathrm{MAX}}$, cyclists could opt for keeping a constant knee power and pedal force production, associated with an increase in tendon excursion and a constant fascicle length. In conclusion, increases in power output lead to greater variations in knee angle, muscle-tendon unit length, tendon length, and pennation angle of vastus lateralis for a similar knee extensor activation and smaller pedal force changes in cyclists from Cluster \#2 compared to Cluster \#1.
\end{abstract}

Keywords: Muscle architecture, muscle-tendon unit, EMG, pedal forces and knee. 


\section{INTRODUCTION}

Maximization of endurance performance involves increasing maximal oxygen uptake $\left(\dot{\mathrm{VO}}_{2 \mathrm{MAX}}\right)$ and sustaining the largest possible power output (PO) in long duration trials. This could be achieved by high intensity interval training with focus at the anaerobic component of training ${ }^{1}$, or by the increase in oxygen consumption and power output corresponding to the second ventilatory threshold ( $\left.\mathrm{PO}_{\mathrm{VT} 2}\right)$. These two strategies might result in increased performance in endurance athletes ${ }^{2}$. Thus, maximal oxygen uptake and anaerobic threshold have both been traditionally used in laboratory testing to predict the potential performance of cyclists and to define training zones ${ }^{2}$.

The comparison between low and high intensity aerobic cycling power outputs involves alterations in leg motion and joint kinetics ${ }^{3}$ and it is likely that cyclists have more than a single strategy to overcome increases in power output during pedalling ${ }^{4}$. Although intuitive, this is critical given that existing recommendations for pedalling technique for cyclists are conflicting 5 . It is possible that some cyclists will benefit from focusing on "circling technique" (i.e. driving forces perpendicular to the crank during pedalling) while others may benefit from focusing on "pushing down" the pedals in the propulsive phase of the crank cycle (i.e. $0-180^{\circ}$ ).

Previous research has shown that increments in power output led to higher activation of lower limb muscles ${ }^{6-8}$ and increased pedal force application ${ }^{9,10}$. However, most studies were conducted at fixed power output (i.e. same power output for all cyclists), which would result in different exercise intensities for each cyclist. Therefore, it appears more relevant to compare power output using intensity-based workloads (i.e. power output relative to a given effort) rather than fixed power output. This approach is helpful for the comparison of athletes when it is done at the power output matched to their physiological responses during racing and training 11,12 . Although increases in fixed power output should be associated to greater muscle activation and pedal force application and/or to greater pedal force effectiveness, power output defined from intensity-based methods should be used to assess cyclists' strategies to accommodate changes in power output for a given exercise effort.

The magnitude of power output for a given pedalling cadence seems to affect the length of active skeletal muscle structures (i.e. fascicle length) during cycling ${ }^{13}$. However, there are no studies looking at the effects of power output on muscle-tendon unit length (e.g. length of active and passive muscle structures) in cyclists to determine the potential elastic components length changes (i.e. tendons and aponeuroses). Austin et al. ${ }^{13}$ used supra maximal $(750 \mathrm{~W})$ in comparison to sub maximal aerobic power output (100 and $250 \mathrm{~W})$. With these large variations in power applied to the pedal, large variations in fascicle length and/or muscle-tendon unit length (e.g. vastus lateralis muscle) are expected to occur. Apart from Austin et al. ${ }^{13}$, the only study in this topic was conducted with non-athletes and limited to a low power output ( 100 W) and a $40 \mathrm{rpm}$ cadence ${ }^{14}$, which are considerably lower than those observed for experienced cyclists. Taken together, these limitations make it unclear if trained cyclists choose a single strategy in terms of activating their muscles, changing the length of the muscle-tendon unit contractile (muscle fascicle length) and elastic components (tendon length), and power production at the knee joint to pedal at the power output of their anaerobic ventilatory threshold compared to their maximal aerobic power output.

To date, there is no clear evidence describing how muscle structure and function (i.e. muscle architecture, muscle-tendon unit length, muscle activation and muscle force) change in cyclists when pedalling at their anaerobic ventilatory threshold power output ( $\mathrm{PO}_{\mathrm{VT} 2}$ ) compared to pedalling at their maximal aerobic power output ( $\mathrm{PO}_{\mathrm{MAX}}$ ). Moreover, different strategies may be employed by cyclists to achieve this goal in terms of motion pattern (e.g. differences in joint kinetics and kinematics). Therefore, the aim of this study was to employ cluster analysis to assess if cyclists would opt for different strategies in terms of neuromuscular patterns (i.e. 
changes in muscle architecture, muscle-tendon unit length, tendon length, muscle activation and pedal forces) when cycling at their $\mathrm{PO}_{\mathrm{VT} 2}$ compared to cycling at their $\mathrm{PO}_{\mathrm{MAX}}$.

\section{METHODS}

\section{Subjects}

Twenty athletes with competitive experience (11 cyclists and 9 triathletes) volunteered for this study. Athletes were $28 \pm 7.3$ years old, with a body mass of $73 \pm 8.0 \mathrm{~kg}$; standing height of $180 \pm 8.1 \mathrm{~cm} ; \mathrm{PO}_{\mathrm{MAX}}$ of $380 \pm 37 \mathrm{~W}$; POMAX/body mass of $5.2 \pm 0.4 \mathrm{~W} / \mathrm{kg}$; maximal oxygen uptake $\left(\dot{\mathrm{VO}}_{2 \mathrm{MAX}}\right)$ of $62 \pm 5.8 \mathrm{ml} / \mathrm{kg} / \mathrm{min}$; power output during the second ventilatory threshold (POVT2): $318 \pm 45 \mathrm{~W}$; and oxygen uptake during the second ventilatory threshold (نंOvT2): 50 $\pm 5.7 \mathrm{ml} \cdot \mathrm{kg}^{-1} \cdot \mathrm{min}^{-1} 15$. Before the evaluation session, all procedures were presented to the participants who signed a consent form to participate in the study that was approved by the Ethics Committee of Human Research where the study was conducted (number 17684).

\section{Protocol}

During the first evaluation session, anthropometrics (standing height and body mass) were measured according to International Society for the Advancement of Kinanthropometry protocols Fel! Ogiltig hyperlänkreferens.. Athletes' femur length (used for fascicle length normalization) was measured from the greater trochanter to the lateral femoral condyle with participants in ventral decubitus. Afterwards, participants warmed-up at $150 \mathrm{~W}$ for $10 \mathrm{~min}$ before the test began. They were assessed using their own bicycles mounted on a stationary cycling trainer (Computrainer, ProLab 3D, Racermate Inc., Seattle, WA, USA) to determine $\mathrm{PO}_{\mathrm{MAX}}$ and $\dot{\mathrm{V}} \mathrm{O}_{2 \mathrm{MAX}}$. The protocol started with $100 \mathrm{~W}$ and was followed by step increments of $25 \mathrm{~W}$ every minute until exhaustion. Pedalling cadence was visually controlled close to $90 \pm 2$ rpm for all athletes using feedback from the cycle trainer head unit (Computrainer, ProLab 3D, Racermate Inc., Seattle, WA, USA). All athletes were instructed to keep their hands on the brake hoods and to remain seated during the test. Oxygen uptake was measured by an opencircuit gas exchange system (CPX/D, Medical Graphics Corp., St Louis, USA). Prior to the incremental test, the $\mathrm{O}_{2}$ and $\mathrm{CO}_{2}$ analysers were calibrated using medical grade gases of known concentration that spanned the physiological range. Gas exchange data were analysed to define the $\mathrm{VO}_{2 \mathrm{MAX}}$ and second ventilatory threshold $\left(\mathrm{VT}_{2}\right)$, following descriptions from Weston and Gabbett ${ }^{16}$.

During the second evaluation session, athletes warmed-up at $150 \mathrm{~W}$ for $10 \mathrm{~min}$ before the test began. They rode 1-min with a cadence of $90 \pm 2 \mathrm{rpm}$ at maximal power output (POMAX) from the incremental test. After two minutes of rest, cyclists performed one 1-min trial with the power output obtained from their second ventilatory threshold (POVT2) at a cadence of $90 \pm 2$ rpm. Force applied on the right pedal, lower limb muscle activation, joint kinematics and muscle architecture from their right vastus lateralis were assessed. The data were collected during the last $20 \mathrm{sec}$ for each trial.

\section{Data collection}

Normal and anterior-posterior components of the force applied on the right pedal were measured using a 2D pedal dynamometer ${ }^{17}$. Pedal forces were recorded at $600 \mathrm{~Hz}$ per channel employing a 16-bit analog-to-digital converter (DI220, Dataq Instruments, USA) using WINDAQ $^{\circledR}$ software (WINDAQ, DataQ Instruments Inc., USA).

Kinematics were determined from images acquired from the right lower limb using one camera four meters from the movement sagittal plane (AVT PIKE F-032, Allied Vision Technologies 
$\mathrm{GmbH}$, Germany), recording at $60 \mathrm{~Hz}$ via AVT ActiveCam viewer software (Allied Vision Technologies $\mathrm{GmbH}$, Germany). Landmarks for the hip, knee and ankle joint axes were defined using reflective markers placed on the participants' right side at the anterior superior iliac spine, greater trochanter, lateral femoral condyle, lateral malleolus, anterior and posterior portions of the pedal. Two markers were taped to the bicycle frame at a known distance from each other and orthogonal to the line of the camera view for linear calibration purposes.

Muscle activity was measured by surface electromyography (EMG) for the vastus medialis, rectus femoris and biceps femoris muscles using a Bortec electromyography system (Octopus AMT-8, Bortec Electronics Inc., Calgary, Canada). Pairs of Ag/AgCl electrodes Meditrace 100 (Kendall Meditrace, Chicopee, Canada) were positioned on the skin in a bipolar configuration with an interelectrode distance of $22 \mathrm{~mm}$ after carefully shaving and cleaning the area using an abrasive cleaner and alcohol swabs to reduce the skin impedance. The electrodes were positioned over the muscle bellies, one third of the muscle length proximal to the midpoint in line with the muscle fibers and taped to the skin using micropore tape (3M Company, USA). The reference electrode was placed over an electrically neutral bony prominence (anterior surface of the tibia). The electrodes' wires were taped to the skin to reduce movement artefact. EMG data were sampled at $2400 \mathrm{~Hz}$ per channel using a 16-bit analog-to-digital converter (DI720, Dataq Instruments, USA) using WINDAQ ${ }^{\circledR}$ software (WINDAQ, DataQ Instruments Inc., USA).

The video with ultrasonography images was acquired from the right vastus lateralis using a probe $(60 \mathrm{~mm}$ linear arrangement, $7.5 \mathrm{MHz}$ ) connected to an ultrasound system (ALOKA, SSD 4000 , Tokyo, Japan). The probe was positioned longitudinal to the muscle belly at $50 \%$ of thigh length ${ }^{18}$. A micropore tape marker was attached to the skin and used as a reference for the position of the probe in relation to the skin. A DVD recorder (R130/XAZ, $30 \mathrm{~Hz}$ Samsumg Inc., Seoul, South Korea) was used to record images at $30 \mathrm{~Hz}$ for offline analysis. All data collection systems (pedal forces, kinematics, EMG and ultrasound) were synchronized by an external trigger as previously described by Muraoka et al. ${ }^{14}$.

\section{Data analysis}

Force and EMG signals were initially time-synchronized off-line to kinematics and ultrasound data using custom made scripts written in MATLAB ${ }^{\circledR}$ (MathWorks Inc., Natick, MA, USA).

Force signals were filtered using a third order zero lag low-pass Butterworth digital filter with a cut-off frequency of $10 \mathrm{~Hz}$. The resultant pedal force was determined from the normal and tangential (anterior-posterior) force components ${ }^{10}$. Kinematic videos were digitized using automatic tracking of markers in Skill Spector software (Video4Coach, Denmark) to obtain Xy coordinates over time. Kinematics were smoothed with a digital second order zero lag lowpass Butterworth filter with a cut-off frequency of $5 \mathrm{~Hz}$. Joint angles of the hip (relative to the horizontal), knee, and ankle were calculated from the smoothed $x-y$ coordinate data. Vastus lateralis, muscle-tendon unit lengths (normalized to femur length; fl) were computed using the model described by Hawkins and Hull, ${ }^{19}$ in MATLAB ${ }^{\circledR}$.

The raw EMG signals were filtered using a fifth order band-pass Butterworth filter with cut-off frequencies of 20 (high pass) and 500 (low pass) Hz. The root mean square (RMS) envelopes $(40 \mathrm{~ms})$ from each muscle of each athlete were then time normalized to the full crank cycle (i.e. 360 samples) and amplitude normalized by their mean values at the POMAX trial.

The right lower limb was modelled as a three-segment rigid body system (thigh, shank and footpedal) with segment mass and centre of mass estimated according to de Leva ${ }^{20}$. Linear and angular velocities and acceleration of the segments' centres of mass were computed from smoothed kinematic data by a three point derivative method ${ }^{21}$. Pedal force components were 
then converted (using pedal angle relative to the horizontal) from their local coordinate system to a global coordinate system. A conversion from the global to the crank coordinate system was later conducted to compute the effective (crank tangential) and radial forces in order to compute the index of effectiveness ${ }^{22}$. Conventional inverse dynamics were conducted to calculate the net joint moments at the ankle and at the knee joints ${ }^{23}$ using adapted scripts of van den Bogert and De Koning ${ }^{24}$. Quadriceps muscle force was estimated by the ratio between the knee extensor moment and the moment arm of the patellar tendon ${ }^{25}$, only when the net knee moment was an extensor moment. Quadriceps force was assumed to be zero when the knee moment was a flexor moment.

Video files of ultrasound data were extracted from the DVD's for visual analysis of the external trigger and edited in Virtual Dub (Avery Lee, USA). Frames were manually digitized by two experienced raters to assess the architectural parameters within each frame using motion analysis software (Skill Spector, Video4Coach, Denmark). Muscle thickness was defined as the average distance in the $y$-axis of the image between the superficial and the deep aponeuroses. Pennation angle was computed by the average angle from the markers digitized on the fascicle and the deep aponeurosis. Fascicle length was computed as shown in equation $1^{13}$ and tendon length was calculated as shown in equation $2^{19}$.

$$
F L=M T / \sin P A
$$

Equation 1. Fascicle length (FL) computed as the ratio between muscle thickness (MT) and the sin of pennation angle (PA). For between-subjects comparisons, fascicle length was normalized to athletes' femur length. All muscle architecture analysis was conducted using custom written scripts in MATLAB ${ }^{\circledR}$.

$$
T L=M T U-(F L * \cos P A)
$$

Equation 2. Tendon length (TL) computed by the subtraction of muscle-tendon unit length (MTU) from the product of fascicle length (FL) and the cosine of fascicle pennation angle (PA).

One virtual marker determined from the anterior and posterior pedal stick markers was created to define the pedal spindle and was used to separate all signals into ten consecutive crank cycles for time normalization. All variables were then averaged for their values during the propulsion phase of the crank cycle $\left(0^{\circ}\right.$ to $\left.180^{\circ}\right)$.

\section{Statistical Analysis}

A cluster analysis was employed to determine performance profiles within the assessed cyclists. Two clustering approaches were used to increase confidence in the stability of the emergent profiles: (i) hierarchical cluster analysis using Ward's linkage method with the squared Euclidean distance measure to provide guidance as to the number of clusters represented in the data; (ii) k-means (nonhierarchical) cluster analysis. Hierarchical dendrogram and agglomeration coefficients were also used to determine the optimal number of clusters. Standardized z scores (i.e., normalization process allowing the comparison of datasets with different units and/or magnitudes) of the differences between the POMAX compared to POVT2, oxygen uptake, resultant force, effective force, index of effectiveness, knee power, knee torque, knee angle, muscle thickness, pennation angle, fascicle length, tendon length, muscle-tendon 
unit length and muscle activation (VM, RF and $\mathrm{BF}$ ) were used in the cluster analysis. Clusters

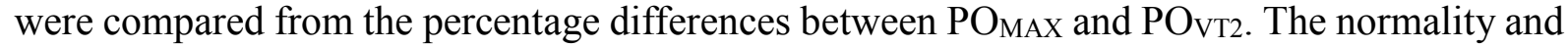
homoscedasticity assumptions were assessed using Shapiro Wilk and the Levene tests, respectively. An independent student's t-test was used to test for differences between Clusters $\# 1$ and \#2. The mean and standard deviation (SD) for each cluster were computed for all variables. All tests were conducted in a statistical package (Stata 12.1, StataCorp LP, USA) with a conventional significance level of $p<0.05$. The cluster analysis was repeated several times, removing each variable one by one. A variable was considered significantly discriminative when the composition of the dendrogram (number of cluster and classification of the subject in the cluster) did not change in comparison to the initial result. The effect size was computed with Cohen's $d$ for the t-test. It was considered a large effect size if $|\mathrm{d}|>0.8^{26}$.

\section{RESULTS}

Athletes were divided into two groups after the cluster analysis, Cluster \#1 with 14 athletes and Cluster \#2 with 6 athletes based on their within-groups similarities. In this line, there were no differences between Clusters \#1 and \#2 for age, body mass, height, $\mathrm{PO}_{\mathrm{MAX}}$, POVT2, or $\dot{\mathrm{VO}}_{2 \mathrm{MAX}}$ (Table 1).

\section{$<<$ Table 1 >>}

Athletes from both Clusters \#1 and \#2 presented similar relative differences between power outputs (PO; $-19 \%$ to $-19 \%$, respectively, $\mathrm{p}=0.70)$, and oxygen uptake $\left(\dot{\mathrm{VO}}_{2} ;-23 \%\right.$ to $-21 \%$ respectively, $\mathrm{p}=0.70)$, as shown in Table 2 . However, Cluster \#1 had greater decreases in resultant force $(-14 \%$ to $-2 \%$, respectively, $\mathrm{p}=0.02)$, effective force $(-12 \%$ to $-2 \%$, respectively, $\mathrm{p}=0.02)$, knee power $(-14 \%$ to $4 \%$ respectively, $\mathrm{p}<0.01)$ and knee torque $(-15 \%$ to $1 \%$, respectively, $\mathrm{p}<0.01)$ compared to Cluster \#2. There were no differences for the index of effectiveness between Clusters \#1 and \#2 (1\% to 1\%, respectively, $\mathrm{p}=0.73$ ).

\section{$<<$ Table 2>>}

For the comparison of joint angle and neuromuscular variables between POVT2 and POMAX, Cluster \#1 presented less variation (Table 3 ) for knee angle ( $1.5 \%$ to $-4 \%$, respectively, $\mathrm{p}<0.01$ ), muscle-tendon unit length (MTU; $0.3 \%$ to $-0.6 \%$, respectively, $\mathrm{p}<0.01)$, pennation angle $(-10 \%$ to $-21 \%$, respectively, $\mathrm{p}=0.04)$, and tendon length $(-0.0 \%$ to $-4.2 \%$, respectively, $\mathrm{p}<0.01)$ compared to Cluster \#2. However, Clusters \#1 and \#2 did not differ for muscle thickness ($3.3 \%$ to $-0.4 \%$, respectively, $\mathrm{p}=0.52)$, fascicle length ( $12 \%$ to $18 \%$, respectively, $\mathrm{p}=0.39)$, and activation of rectus femoris (RF; $-14 \%$ to $-5 \%$, respectively; $\mathrm{p}=0.38$ ), vastus medialis (VM; $20 \%$ to $-17 \%$, respectively, $\mathrm{p}=0.64$ ) and biceps femoris $(\mathrm{BF} ;-8 \%$ to $-14 \%$, respectively, $\mathrm{p}=0.46)$.

\section{$<<$ Table 3>>}

\section{DISCUSSION}

The purpose of this study was to employ cluster analysis to assess if cyclists would opt for different strategies in terms of neuromuscular patterns (i.e. changes in muscle architecture, muscle-tendon unit length, tendon length, muscle activation and pedal forces) when cycling at their POVT2 compared to cycling at their $\mathrm{PO}_{\mathrm{MAX}}$. This method resulted in two Clusters of cyclists with different techniques when comparing a submaximal (POvт2) to a maximal power output 
( $\mathrm{PO}_{\mathrm{MAX}}$ ). Cyclists from Cluster \#1 presented larger resultant and effective pedal force application at the crank cycle propulsive phase than the Cluster \#2 cyclists. These results were followed by greater knee power for Cluster \#1 along with smaller variations in knee flexion, muscle-tendon unit length, fascicle length, pennation angle and tendon length than Cluster \#2. Taken together, these findings suggest differences in pedalling technique enforced by different muscle function between Cluster \#1 compared to Cluster \#2.

When comparing the differences between $\mathrm{PO}_{\mathrm{VT} 2}$ to $\mathrm{PO}_{\mathrm{MAX}}$, cyclists from Cluster \#1 relied on larger knee power while cyclists from Cluster \#2 opted for reducing knee power. This smaller knee power output was impacted by greater knee flexion angle in Cluster \#2 compared to Cluster \#1 leading to greater pennation angle and longer tendon length. Larger increases in knee joint angle (i.e. greater knee flexion) were observed for Cluster \#2, which led to larger increases in VL muscle-tendon unit length. However, VL fascicle length shortening was similar between clusters. These results indicate that fascicle length changes were similar between power outputs (sub maximal vs. maximal effort). Moreover, non-contractile elements (i.e. tendons and aponeuroses) would need to lengthen in order to provide additional stress in muscles to sustain potentially larger muscle forces at the higher power output. These smaller changes in knee power for a similar fascicle length alterations of Cluster \#2 compared to Cluster \#1 suggest that Cluster \#2 presented better neuromuscular efficiency than the latter. The combined increase in pennation angle and tendon length (larger in Cluster \#2) might be associated with a sarcomere length optimization, maintaining the contracting length closer to the optimal length for force production (i.e. facilitating the greatest possible number of established cross-bridges) at higher power output levels ${ }^{13}$. These architectural modifications were probably related to an increase in the length of the muscle's passive elements (series elastic elements) for a constant muscletendon unit length. However, this hypothesis cannot be tested based on our results but should be investigated in future studies.

Reduced knee power for cyclists in Cluster \#2 resulted in lower force applied to the pedal than observed in Cluster \#1. During the propulsive phase of the crank cycle, cyclists have the option to produce the same power by applying large pedal force or by driving pedal forces to optimize effective force (i.e. force tangential to the crank) and crank torque, therefore increasing their pedalling effectiveness ${ }^{27}$. Although cyclists from Cluster \#2 presented lower effective force than their counterparts, this was potentially due to lower total pedal force application, given the index of effectiveness (ratio between tangential and radial crank forces) did not differ between clusters. It may be the case that cyclists from Cluster \#2 had enhanced pedal pulling forces during the recovery phase, which was not explored in our study. Previous findings also highlighted that cyclists may increase hip and knee flexion torques without increasing the index of effectiveness ${ }^{3,28}$. Therefore, we can infer that cyclists from Cluster \#1 opted for a technique linked to larger knee power production and Cluster \#2 opted for applying more power through their hip or ankle joints during the propulsive phase of crank cycle.

The larger efforts required by the knee joint muscles from cyclists in Cluster \#1 would be expected to lead to greater neural drive (i.e. enhanced activation), which was not confirmed. Both groups presented similar increases in knee extensors recruitment (i.e. vastus medialis and rectus femoris) and flexors (i.e. biceps femoris) when power output was increased. Similar muscle activation may suggest that neural drive is controlled to sustain a given fascicle length (as previously discussed) and a similar energy cost. Given that the power output levels used in our study were individually selected from $\mathrm{VT}_{2}$, individual relative energy cost should have been consistent among groups. That adds to the similar between-groups muscle recruitment given muscle activation is linked to power output increase in cycling ${ }^{29}$. Increased mechanical requirements for pedalling at higher power output were linked to an increase in resultant force, effective force, crank power, knee power and higher knee torque in Cluster \#1 compared with 
Cluster \#2. These greater efforts enforced that knee joint muscles were submitted to larger mechanical demands (i.e. Clusters \#1), resulting in larger neuromuscular efficiency (i.e. larger joint power for a similar muscle activation) in Cluster \#2 compared the Cluster \#1. Cyclists in Cluster \#2 were able to generate a similar power output (POMAX and POVT2) with less force variation (knee power and torque) when cycling at a sub maximal compared to a maximal effort level. As muscle activation was similar and changes in muscle architecture and in muscletendon unit length were different between the two clusters, it is fair to say that, for a similar neural drive, Cluster \#2 presented better neuromuscular efficiency compared to Cluster \#1.

A secondary adaptation to higher power output may be associated with changes in pedalling technique by sharing mechanical requirements among different muscles ${ }^{30}$. This may potentially result in different adaptation for cyclists with varying skill levels ${ }^{31}$ or in trained cyclists compared to non-athletes ${ }^{32}$. A comparison of responses between cyclists of different skill levels and disciplines (e.g. track vs. road) could provide important information to assess if differences in training programs could lead to differences in acute adaptation of contractile elements to different effort levels in cycling. In addition, determining the tendon mechanical properties during cycling might help to elucidate if the acute effects of power output on muscle architecture are absorbed by changes in the tendon's structural and mechanical properties.

Our experimental design was limited to some extent. Our data was contained to the right lower limb, which limits any association between power output and pedalling asymmetries. Future studies could shed light on bilateral pedalling technique while cyclists are compared in different workload level. However, the present study showed that there are different adaptations in physiological and biomechanical variables between groups of athletes with similar performance. These adaptations are mainly related to increased tendon length without changes in fascicle length at the Cluster \#2 compared to Cluster \#1. Therefore, longer muscle-tendon unit length, as consequence from changes in knee joint kinematics, was mostly due to alterations in tendon length (not fascicle length). The maximum power output was always used as the first trial for data collection for all athletes, which may have added an order effect to our data. We opted for this sequence in order to gather the maximal effort from athletes without leading to fatigue effects in the $\mathrm{PO}_{\mathrm{MAX}}$ trial. Due to the short duration of this maximal effort (1-min), we do not expect that it could have affected the following submaximal trial (i.e. $\mathrm{VT}_{2}$ ).

\section{PRACTICAL IMPLICATIONS}

Findings from this study provide information on the interaction between active and passive skeletal muscle components. Cyclists and coaches should expect that, during increased power output trials, greater changes in muscle-tendon unit length and tendon length combined with increased pennation angle and fewer differences in fascicle length should be observed among athletes. Results from this study showed that the contribution from skeletal muscles passive components can be larger than expected during cycling. It is yet to be determined if the previously observed smaller eccentric components during cycling ${ }^{33}$ may be larger given the aforementioned mechanism. Implications for training could be linked to an increased need for strength training for cycling ${ }^{34}$.

\section{CONCLUSION}

Clusters analysis identified two predominant clusters among our athletes, which were compared retrospectively. A larger power output led to greater knee angle, muscle-tendon unit length, tendon length, and vastus lateralis pennation angle in cyclists from Cluster \#2, with minor variations in pedal forces during the pedalling cycle propulsion, compared with cyclists from Cluster \#1. However, these changes did not result in differences in muscle activation of knee 
extensors and flexors or in power output and oxygen uptake between the two clusters.

\section{REFERENCES}

1. Laursen PB, Jenkins DG. The scientific basis for high-intensity interval training: optimising training programmes and maximising performance in highly trained endurance athletes. Sports Medicine 2002;32(1):53-73.

2. Joyner MJ, Coyle EF. Endurance exercise performance: the physiology of champions. The Journal of physiology 2008;586(1):35-44.

3. Bini RR, Diefenthaeler F. Kinetics and kinematics analysis of incremental cycling to exhaustion. Sports Biomechanics 2010;9(4):223-35.

4. Kautz SA, Feltner ME, Coyle EF, Baylor AM. The pedaling technique of elite endurance cyclists: changes with increasing workload at constant cadence. Journal of Applied Biomechanics 1991;7(1):29-53.

5. Bini RR, Hume PA, Croft J, Kilding A. Pedal force effectiveness in Cycling: a review of constraints and training effects. Journal of Science and Cycling 2013;2(1):11-24.

6. Bijker KE, de Groot G, Hollander AP. Differences in leg muscle activity during running and cycling in humans. European journal of applied physiology 2002;87(6):556-61.

7. Hug F, Dorel S. Electromyographic analysis of pedaling: a review. Journal of Electromyography Kinesiology 2009;19(2):182-98.

8. MacIntosh BR, Neptune RR, Horton JF. Cadence, power, and muscle activation in cycle ergometry. Medicine and Science in Sports and Exercise 2000;32(7):1281-7.

9. Patterson RP, Moreno MI. Bicycle pedalling forces as a function of pedalling rate and power output. Medicine and Science in Sports and Exercise 1990;22(4):512-6.

10. Rossato M, Bini RR, Carpes FP, Diefenthaeler F, Moro AR. Cadence and workload effects on pedaling technique of well-trained cyclists. International Journal of Sports Medicine 2008;29(9):746-52.

11. Abbiss CR, Quod MJ, Martin DT, Netto KJ, Nosaka K, Lee H et al. Dynamic pacing strategies during the cycle phase of an Ironman triathlon. Medicine and Science in Sports and Exercise 2006;38(4):726-34.

12. Bernard T, Hausswirth C, Le Meur Y, Bignet F, Dorel S, Brisswalter J. Distribution of power output during the cycling stage of a Triathlon World Cup. Medicine and Science in Sports and Exercise 2009;41(6):1296-302.

13. Austin N, Nilwik R, Herzog W. In vivo operational fascicle lengths of vastus lateralis during sub-maximal and maximal cycling. Journal of Biomechanics 2010;43(12):2394-9.

14. Muraoka T, Kawakami Y, Tachi M, Fukunaga T. Muscle fiber and tendon length changes in the human vastus lateralis during slow pedaling. Journal of Applied Physiology 2001;91(5):2035-40.

15. Ansley L, Cangley P. Determinants of "optimal" cadence during cycling. European Journal of Sport Science 2009;9(2):61-85.

16. Weston SB, Gabbett TJ. Reproducibility of ventilation of thresholds in trained cyclists during ramp cycle exercise. Journal of Science and Medicine in Sport / Sports Medicine Australia 2001;4(3):357-66.

17. Nabinger E, Iturrioz, I., Zaro, M. A. Development of a triaxial force platform for the measurement of force at a bicycle pedal. Paper presented at the International Symposium of Biomechanics in Sports, Caceres - Extremadura - Spain from 2002.

18. Blazevich AJ, Gill ND, Zhou S. Intra- and intermuscular variation in human quadriceps femoris architecture assessed in vivo. Journal of Anatomy 2006;209(3):289-310.

19. Hawkins D, Hull ML. A method for determining lower extremity muscle-tendon lengths during flexion/extension movements. Journal of Biomechanics 1990;23(5):487-94. 
20. De Leva P. Adjustments to Zatsiorsky-Seluyanov's segment inertia parameters. Journal of Biomechanics 1996;29(9):1223-30.

21. Winter DA. Biomechanics and motor control of human movement. John Wiley \& Sons 2005.

22. Bini RR, Hume PA. Effects of workload and pedalling cadence on knee forces in competitive cyclists. Sports Biomechanics 2013;12(2):93-107.

23. Redfield R, Hull ML. On the relation between joint moments and pedalling rates at constant power in bicycling. Journal of Biomechanics 1986;19(4):317-29.

24. Van den Bogert AJ, De Koning JJ. On optimal filtering for inverse dynamics. IXth Biennial Conference of Canadian Society for Biomechanics, Vancouver 1996.

25. Herzog W, Read LJ. Lines of action and moment arms of the major force-carrying structures crossing the human knee joint. Journal of Anatomy 1993;182 ( Pt 2):213-30.

26. Cohen J. Statistical power analysis for the behavioral sciences. 2nd edn Lawrence Erlbaum Associates, Hillsdale 1988.

27. Bohm H, Siebert S, Walsh M. Effects of short-term training using SmartCranks on cycle work distribution and power output during cycling. European Journal of Applied Physiology 2008;103(2):225-32.

28. Bini RR, Seger D, Lanferdini FJ, Lopes AL. Joint kinematics assessment during cycling incremental test to exhaustion. Isokinetics and Exercise Science 2012;20(1):99-105.

29. Hug F, Decherchi P, Marqueste T, Jammes Y. EMG versus oxygen uptake during cycling exercise in trained and untrained subjects. Journal of electromyography and kinesiology : official journal of the International Society of Electrophysiological Kinesiology 2004;14(2):187-95.

30. Mornieux G, Gollhofer A, Stapelfeldt B. Muscle coordination while pulling up during cycling. International Journal of Sports Medicine 2010;31(12):843-6.

31. Chapman AR, Vicenzino B, Blanch P, Hodges PW. Patterns of leg muscle recruitment vary between novice and highly trained cyclists. Journal of Electromyography and Kinesiology 2008;18(3):359-71.

32. Mornieux G, Stapelfeldt B, Gollhofer A, Belli A. Effects of pedal type and pull-up action during cycling. International Journal of Sports Medicine 2008;29(10):817-22.

33. Connick MJ, Li FX. The impact of altered task mechanics on timing and duration of eccentric bi-articular muscle contractions during cycling. Journal of Electromyography and Kinesiology Kinesiology 2013;23(1):223-9.

34. Hansen EA, Ronnestad BR, Vegge G, Raastad T. Cyclists' improvement of pedaling efficacy and performance after heavy strength training. International Journal Sports Physiology and Performance 2012;7(4):313-21.

\section{TABLE CAPTIONS}

Table 1. Mean \pm standard deviation values of Clusters \#1 and \#2 for all variables (age, body mass, height, maximal power output, power output of the second ventilatory threshold and maximal oxygen uptake). P-value and effect sizes are shown for the between-clusters comparison.

Table 2. Mean \pm standard deviation values for power output, power output/body mass, oxygen uptake, resultant force, effective force, index effectiveness, knee power and knee torque of Clusters \#1 and \#2 at POMAX, at POvT2 and percentage differences of POVT2 with respect to $\mathrm{PO}_{\mathrm{MAX}}$ for each Cluster group. P-values and effect sizes are also shown for the percentage differences comparison. 
Table 3. Mean \pm standard deviation values for knee angle, muscle architecture (muscle thickness, pennation angle, fascicle length, tendon length, muscle tendon unit length) and muscle activation (vastus medialis, rectus femoris and biceps femoris) of Clusters \#1 and \#2 at $\mathrm{PO}_{\mathrm{MAX}}$, at $\mathrm{PO}_{\mathrm{VT} 2}$ and percentage differences of $\mathrm{PO}_{\mathrm{VT} 2}$ with respect to $\mathrm{PO}_{\mathrm{MAX}}$ for each Cluster group. P-values and effect sizes are also shown for the percentage differences comparison. 
Table 1. Mean \pm standard deviation values of Clusters \#1 and \#2 for all variables (age, body mass, height, maximal power output, power output of the second ventilatory threshold and maximal oxygen uptake). P-value and effect sizes are shown for the between-clusters comparison.

\begin{tabular}{lccc}
\hline \hline & Cluster \#1 & Cluster \#2 & $\begin{array}{c}\text { p-value } \\
\text { (Cohen' d) }\end{array}$ \\
\hline \hline n & 14 & 6 & -- \\
Age (years) & $28.1 \pm 7.9$ & $27.7 \pm 6.2$ & $0.91(0.06)$ \\
Body Mass (kg) & $74.3 \pm 8.9$ & $71.2 \pm 5.5$ & $0.44(0.43)$ \\
Height (cm) & $180.6 \pm 8.6$ & $177.4 \pm 7.0$ & $0.43(0.41)$ \\
POMax (W) & $383.9 \pm 40.0$ & $370.8 \pm 33.2$ & $0.49(0.36)$ \\
POvT2 (W) & $321.5 \pm 39.9$ & $310.7 \pm 59.0$ & $0.63(0.22)$ \\
VO2 & $60.5 \pm 5.6$ & $64.1 \pm 5.8$ & $0.20(0.64)$ \\
\hline \hline
\end{tabular}

$\mathrm{PO}_{\mathrm{MAX}}$ and $\mathrm{PO}_{\mathrm{VT} 2}=$ Mean maximal power output during the crank cycle and mean power output of the second ventilatory threshold during the crank cycle, respectively; $\dot{\mathrm{V}} \mathrm{O}_{2 \mathrm{MAX}}=$ Mean maximal oxygen uptake in the incremental test. 
Table 2. Mean \pm standard deviation values for power output, power output/body mass, oxygen uptake, resultant force, effective force, index effectiveness, knee power and knee torque of Clusters \#1 and \#2 at POMAX, at POVT2 and percentage differences of POVT2 with respect to POMAX for each Cluster group. $\mathrm{P}$-values and effect sizes are also shown for the percentage differences comparison.

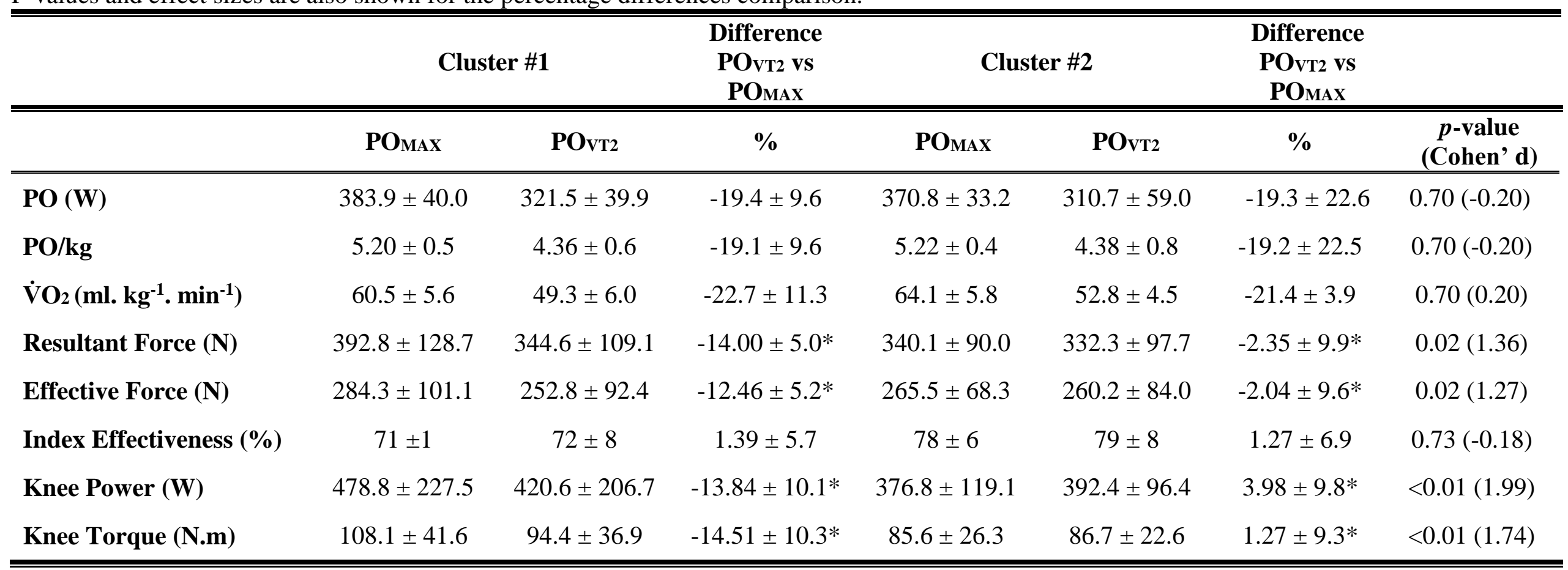

*Indicates significant differences for the between-percentage differences comparison [percentage difference obtained at the power output of the second ventilatory threshold $\left(\mathrm{PO}_{\mathrm{vT} 2}\right)$ relative to the maximal power output (POMAX)] of Clusters \#1 and \#2.

$\mathrm{PO}_{\mathrm{MAX}}$ and $\mathrm{POV}_{2}=$ Mean power output during the propulsion phase of the crank cycle $\left(0-180^{\circ}\right)$ and mean power output of the second ventilatory threshold during the propulsion phase of the crank cycle, respectively.

$\mathrm{PO}(\mathrm{W})=$ Power Output; and $\mathrm{PO} / \mathrm{kg}=$ Power Output/Body Mass and $\dot{\mathrm{VO}}_{2}=$ oxygen uptake volume; $(\mathrm{PO}, \mathrm{PO} / \mathrm{kg}, \dot{\mathrm{VO}} 2$ found during incremental test during the overall crank cycle). 
Table 3. Mean \pm standard deviation values for knee angle, muscle architecture (muscle thickness, pennation angle, fascicle length, tendon length, muscle tendon unit length) and muscle activation (vastus medialis, rectus femoris and biceps femoris) of Clusters \#1 and \#2 at POMAX, at POvT2 and percentage differences of $\mathrm{PO}_{\mathrm{VT} 2}$ with respect to $\mathrm{PO}_{\mathrm{MAX}}$ for each Cluster group. P-values and effect sizes are also shown for the percentage differences comparison.

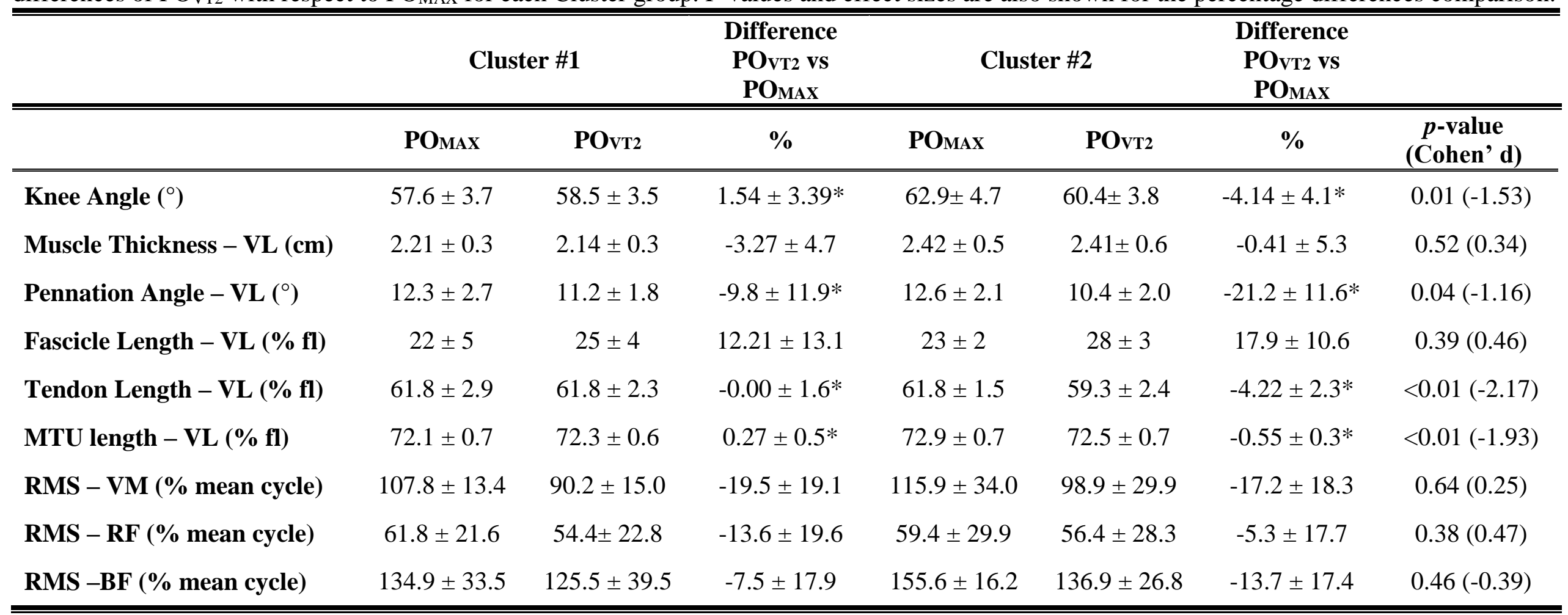

*Indicates significant differences for the between-percentage differences comparison [percentage difference obtained at the power output of the second ventilatory threshold $\left(\mathrm{PO}_{\mathrm{VT} 2}\right)$ relative to the maximal power output $\left.\left(\mathrm{PO}_{\mathrm{MAX}}\right)\right]$ of Clusters \#1 and \#2.

$\mathrm{PO}_{\mathrm{MAX}}$ and $\mathrm{PO}_{\mathrm{VT} 2}=$ Mean power output during the propulsion phase of the crank cycle $\left(0-180^{\circ}\right)$ and mean power output of the second ventilatory threshold during the propulsion phase of crank cycle, respectively; VL = Vastus Lateralis; VM = Vastus Medialis; RF = Rectus Femoris; BF = Biceps Femoris; MTU = Muscle Tendon Unit Length; \% fl = \% of femur's length. 